\title{
Tubule-specific protein nanocages potentiate targeted renal fibrosis therapy
}

\author{
Xuan Zhang ${ }^{1 \dagger}$, Qian Chen ${ }^{2 \dagger}$, Liyuan Zhang ${ }^{1}$, Haiping Zheng ${ }^{3}$, Chunjie Lin ${ }^{4}$, Qunfang Yang ${ }^{1}$, Tao Liu', \\ Haigang Zhang ${ }^{1}$, Xiaohong Chen ${ }^{1}$, Lei Ren $^{5^{*}}$ and Wenjun Shan ${ }^{1,6^{*}}$ (D)
}

\begin{abstract}
Background: Despite the dramatic advances in modern medicine, efficient therapeutic measures for renal fibrosis remain limited. Celastrol (CLT) is effective in treating renal fibrosis in rat models, while causing severe systemic toxicity. Thus, we designed a tubule-specific nanocage (K3-HBC NCs) that effectively deliver CLT to tubular epithelial cell in a virus-like manner. The targeting ligand (K3) to tubular epithelial cells was displayed on the surface of Hepatitis B core protein $(\mathrm{HBC}) \mathrm{NCs}$ by genetic fusion to the major immunodominant loop region. Ultra-small CLT nanodots were subtly encapsulated into the cavity through electrostatic interaction with the disassembly and reassembly of K3-HBC NCs, to yield K3-HBC/CLT complex. The efficacy of K3-HBC/CLT NCs were demonstrated in Unilateral ureteral obstruction (UUO)-induced renal fibrosis.

Results: The self-assembled K3-HBC/CLT could specifically target tubular epithelial cells via affinity with K3 ligand binding to the megalin receptor, significantly attenuating renal fibrosis. Remarkably, K3-HBC/CLT NCs significantly increased therapeutic efficacy and reduced the systemic toxicity in comparison with free CLT in UUO-induced mouse renal fibrosis model. Importantly, analysis of RNA sequencing data suggested that the anti-fibrotic effect of K3-HBC/ CLT could be attributed to suppression of premature senescence in tubular epithelial cells via p21 ${ }^{\text {Cip } 1}$ and p16 $16^{\text {Ink4a }}$ pathway.
\end{abstract}

Conclusion: The tubule-specific K3-HBC/CLT represented a promising option to realize precise treatment for renal fibrosis.

Keywords: Protein nanocage, Celastrol, Target therapy, Cell senescence, Renal fibrosis

\section{Introduction}

Chronic kidney disease (CKD) is a major public health concern worldwide [1]. Renal fibrosis is the common ultimate outcome of CKD and leads to irreversible loss of renal parenchyma and end-stage renal failure where the patients require dialysis or renal transplantation [2].

\footnotetext{
*Correspondence: renlei@xmu.edu.cn; wjshan@tmmu.edu.cn

† Xuan Zhang and Qian Chen contributed equally to this work

${ }^{1}$ Department of Pharmacology, College of Pharmacy and Laboratory

Medicine, Army Medical University (Third Military Medical University), Chongqing 400038, People's Republic of China

${ }^{5}$ Department of Biomaterials, College of Materials, Xiamen University, Xiamen 361005, People's Republic of China

Full list of author information is available at the end of the article
}

So far, fibrotic nephropathy is a severe clinical problem without effective treatment strategies and its pathogenesis remains to be elucidated $[3,4]$.

For a long time, many therapeutic approaches have focused on fibroblasts and myofibroblasts as these cells are the effectors of renal fibrosis [5]. However, these treatments have been disappointing. A number of important recent advances have clarified that the injured tubular epithelial cell is an important orchestrator in the process of renal fibrosis [6]. Tubular epithelial cells are essential for injury. They can adopt different repair mechanisms to survive injury and recover normal kidney function. But as the injury is severe and persistent, normal regeneration mechanisms may not work, and tubular epithelial 
cells might undergo maladaptive repair, which promote tubular atrophy and exacerbate renal fibrosis. The maladaptive tubular epithelial cells can be transformed into a secreted phenotype, thereby generating and releasing various biologically active molecules to precipitate the recruitment of inflammatory cells, the activation of fibroblasts and the loss of endothelial cells, which ultimately drives tubulointerstitial inflammation and fibrosis [7]. Furthermore, tubular epithelial cells can directly contribute to renal fibrosis via epithelial-mesenchymal transition (EMT), a phenotypic conversion program [8]. Tubular epithelial cells could be a promising novel target for ameliorating or even reversing renal fibrosis, but have received considerably less attention.

Natural products play a critical role in finding potential therapeutic agents [9]. For example, Tripterygiumwilfordii Hook F (TWHF), a traditional Chinese medicine, has been widely used in CKD in China for a long time [10], due to its strong anti-inflammatory and immunosuppressive effects [11]. Celastrol (CLT) is one of the predominantly active products in TWHF formulations and has the potential to protect kidney structure and function during chronic kidney injury. However, CLT was reported to induce severe cardiotoxicity, hepatotoxicity, neurotoxicity and reproductive disorder after systemic administration [12]. Meanwhile, poor water solubility $\left(13.25 \pm 0.83 \mu \mathrm{g} \mathrm{mL} \mathrm{m}^{-1}\right.$ at $\left.37^{\circ} \mathrm{C}\right)$, low bioavailability and narrow window of dosage also restricted its further therapeutic application [13]. According to these rationales, targeted delivery of potentially therapeutic CLT to tubular epithelial cells could be an effective antifibrotic strategy.

Natural nanoparticles such as viruses possess their own delivery mechanisms that bear a striking resemblance to the action of many drug delivery vectors [14]. They can cross the human biological barrier easily through specific interactions with target cells. Protein nanocages (NCs) that mimic the overall structure and features of authentic virus without containing viral genetic materials are attractive drug delivery platforms [15]. They are formed by the natural self-assembly of protein subunits, and their repetitive surfaces can be bioengineered by specific ligand to recognize target cell [16]. Their cavity inside the particles can be used to encapsulate therapeutic agents. Moreover, chemical and genetic modifications of NCs can converge the advantageous properties to improve the therapeutic efficiencies.

Herein, we designed a biomimetic nanosystem that could precisely deliver CLT to tubular epithelial cells via a virus-like mechanism. We prepared the bioengineered Hepatitis B core protein ( $\mathrm{HBc}$ ) NCs to specifically recognize tubular epithelial cells. The peptide sequence of (KKEEE)3 (K3), a targeting ligand to tubular epithelial cells [17], was exposed on the surface of the nanocage (K3-HBc NCs). Ultra-small CLT nanodots were subtly encapsulated into the cavity through electrostatic interaction with the disassembly and reassembly of K3-HBc $\mathrm{NCs}$, to yield K3-HBc/CLT complex. The resultant particles thus gain a uniform structure, controlled selfassembly, well biocompatibility and biodegradability, as well as high specificity. Therefore, the present complex achieved high-efficient targeted therapy for renal fibrosis (Scheme 1).

\section{Results}

\section{CLT attenuates unilateral ureteral obstruction} (UUO)-induced renal fibrosis with severe toxicity

We first studied the therapeutic effect of CLT. In the UUO group, CLT $\left(1 \mathrm{mg} \mathrm{kg}^{-1}\right)$ was administered by intraperitoneal injection every other day. The ratio of kidney weight to body weight (kidney index) after 2 weeks treatment was shown in Additional file 1: Figure S1A. The left kidney index was $4.23 \pm 0.05 \%$ in sham group, whereas it increased to $6.19 \pm 0.86 \%$ in $\mathrm{UUO}+0.9 \% \mathrm{NaCl}$ group. However, treatment with CLT reduced the left kidney index to $5.91 \pm 0.39 \%$. Since all the right kidney in each group was not obstructed, there was no significant change in the right kidney index. Increased kidney index implied loss of kidney function, treatment with CLT might rescue some of the negative impact of UUO on kidney function. The hematoxylin and eosin (H\&E) staining revealed that CLT-treated rats had less fibrotic changes in the obstructed kidney than $0.9 \%-\mathrm{NaCl}$-treated rats (Additional file 1: Figure S1B). Renal fibrosis was much attributed to excess deposition of extracellular matrix components such as collagen [2]. We then examined the collagen deposition in renal tissue using Masson's trichrome staining. While the collagen deposition increased significantly in the rats subjected to UUO, a marked reduction was detected in CLT-treated rats (Additional file 1: Figure S1C). The fibrotic area represented by Masson's trichrome staining in each group was analyzed in Additional file 1: Figure S1D. The fibrotic area value was $5.54 \pm 0.82$ in sham group, compared with a robust elevation to $183.29 \pm 7.98$ in $\mathrm{UUO}+0.9 \% \mathrm{NaCl}$ group. We found that CLT could decrease this value to $17.06 \pm 3.22$, implying that more than $90 \%$ collagen was eliminated. $\alpha$-smooth muscle actin ( $\alpha$-SMA), a major morphological characteristic of myofibroblasts, plays a crucial role in the development and progression of renal tubulointerstitial fibrosis [18]. In Additional file 1: Figure S1E and F, the extremely high expression level of $\alpha$-SMA in UUO $+0.9 \%$ $\mathrm{NaCl}$ group was decreased from $154.98 \pm 34.10$ (fold of sham group) to $41.16 \pm 8.39$ by CLT. The immunostaining of $\alpha$-SMA in the renal tissue, as well as another two important fibrotic markers including transforming 


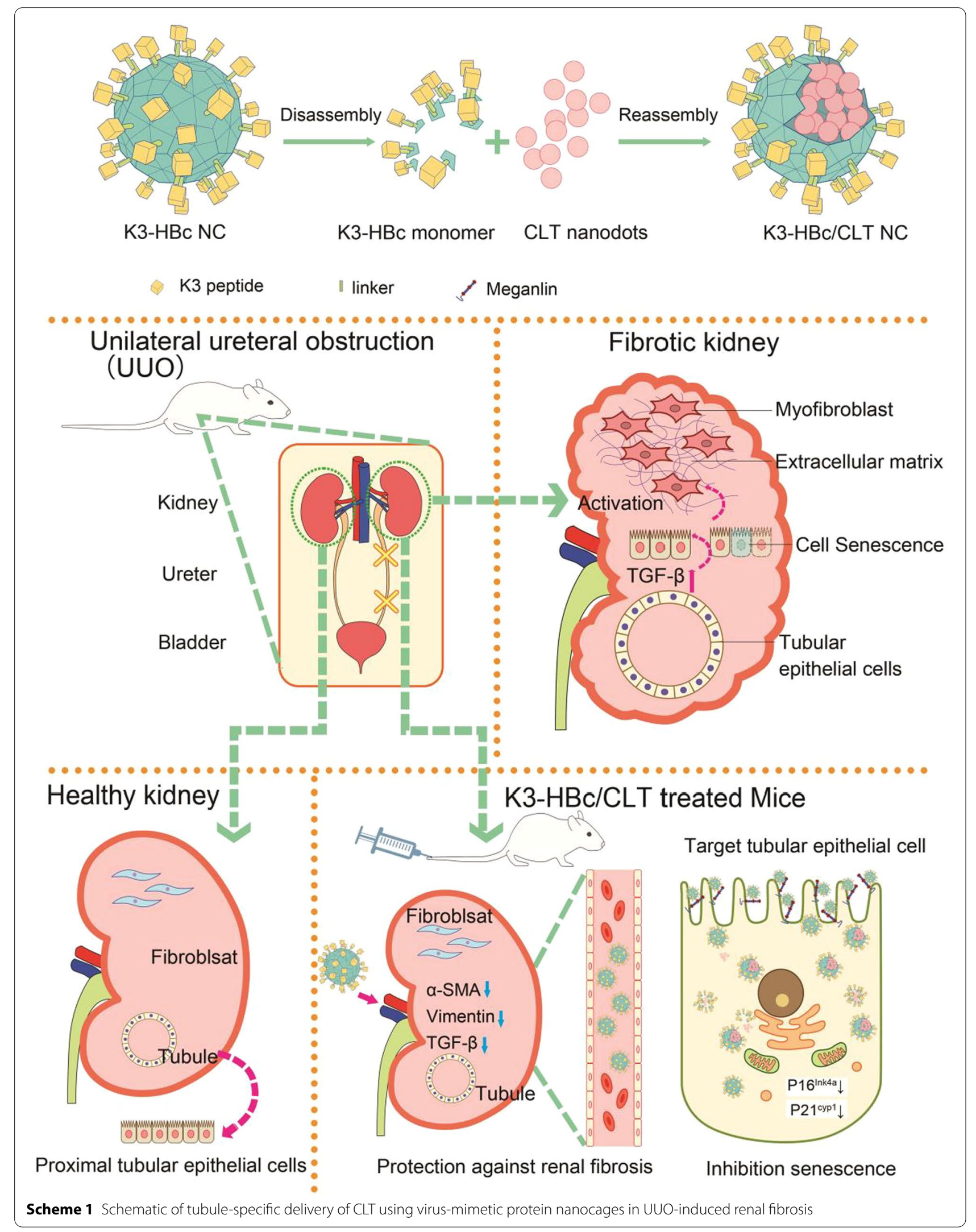


growth factor-beta (TGF- $\beta$ ) and collagen I, was further carried out. Similarly, elevated $\alpha$-SMA expression (Additional file 1: Figure S1G and $\mathrm{H}$ ) in the UUO group and its decrease in response to CLT was confirmed. Among a panel of fibrogenic factors, TGF- $\beta$ is considered a key regulatory molecule in renal fibrotic process [19]. Although TGF- $\beta$ was expressed at highly elevated levels in the renal tubular epithelial cells circulating around the tubules in $\mathrm{UUO}+0.9 \% \mathrm{NaCl}$ group (Additional file 1: Figure S1G), the integrated optical density (IOD) of TGF- $\beta$ was decreased from $55.46 \pm 6.29$ to $15.94 \pm 3.22$ by CLT (Additional file 1: Figure S1I). The collagen I expressions was significantly lower in UUO + CLT group than in $\mathrm{UUO}+0.9 \% \mathrm{NaCl}$ group (Additional file 1: Figure S1G, J). These findings suggested that CLT harbored a potential therapeutic effect on renal fibrosis. However, the systemic toxic effects caused by CLT were inevitable. The rats with free CLT had severe weight loss, while the rats in other groups appeared normal (Additional file 1: Figure S1K). Moreover, serious perivisceral adhesion was found in the healthy rats after administration of free CLT. The toxic effect of CLT on major organs such as heart, liver, spleen, lung, and brain were further examined by H\&E staining (Additional file 1: Figure S2). Especially, myofibrillar damage, cellular atrophy in heart samples, dilatation of blood sinus in the liver and pyknosis of neurons in brain were observed. Taking together, CLT showed extraordinary protective effect against renal fibrosis while accompanied by severe toxicity effects.

\section{Characterization of K3-HBc/CLT NCs}

We next tried to develop virus-mimetic protein nanoparticles derived from $\mathrm{HBc}$ NCs for targeting delivery of CLT. HBc NC is the most flexible and promising model for medical research among various types of protein NCs $[20,21]$. To trigger exceptional renal specificity at high accumulation rates, we generated the $\mathrm{K} 3-\mathrm{HBc} \mathrm{NCs}$ by inserting $\mathrm{K} 3$ peptide (flanked by two glycine-rich flexible linkers) into the surface-exposed spike of $\mathrm{HBc} \mathrm{NC}$, and used $E$. coli as a natural bio-factory to produce bioengineered K3-HBc proteins. SDS-PAGE analysis showed the bands corresponding to the indicated molecular weight of $\mathrm{HBc}-183$ and $\mathrm{K} 3-\mathrm{HBc}$ monomer, respectively (Additional file 1: Figure S3A). Western blot analysis using anti- $\mathrm{HBc}$ as primary antibody was conducted to validate intrinsic $\mathrm{HBC}$ immunogenicity of $\mathrm{K} 3-\mathrm{HBc}$ protein. As shown in Additional file 1: Figure S3B, anti-HBc antibody could recognize $\mathrm{HBc}-183$ but not K3-HBc, indicating the inserted $\mathrm{K} 3$ peptide replaced the epitope ofthe MIR region. Meanwhile, CLT nanodots with a diameters of $13.2 \pm 2.1$ (Fig. 1A, B) had been successfully prepared using a brilliant droplet-confined/cryodesiccation-driven crystallization approach [22]. The zeta potential of CLT nanodots was $-21.7 \mathrm{mV}$, indicating the negatively charged surface of CLT nanodots (Additional file 1: Figure S4). The retained arginine-rich domain of $\mathrm{HBC}$ protein provided a positively charged microenvironment inside the nanoparticle of $\mathrm{K} 3-\mathrm{HBc} \mathrm{NCs}$, which facilitated encapsulation of negatively charged CLT nanodots through electrostatic interactions. As a result, CLT nanodots could be efficiently loaded into the cavities through the controlled disassembly and reassembly of K3-HBc NCs. Negatively stained transmission electron microscope (TEM) confirmed monodispersed and well-defined spherical particles of K3-HBc NCs (Fig. 1A, B). Thus incorporation of $\mathrm{K} 3$ peptide to the spike of $\mathrm{HBc}$ seemed to neither suppress $\mathrm{K} 3-\mathrm{HBc}$ expression nor interfere their self-assembly. The TEM images of the assemble mixture containing $\mathrm{K} 3-\mathrm{HBc}$ subunits and CLT nanodots were shown in Fig. 1A, B. This result demonstrated that ultra-small CLT nanodots were successfully encapsulated into the $\mathrm{K} 3-\mathrm{HBc} \mathrm{NCs}$. The payload was determined to be $1027 \pm 39$ CLT molecules per one nanocage using UVvis spectrophotometer. Dynamic Light Scattering (DLS) analysis conducted on $\mathrm{K} 3-\mathrm{HBc} \mathrm{NCs}$ and $\mathrm{K} 3-\mathrm{HBc} / \mathrm{CLT}$ NCs yielded hydrodynamic diameters of $35.3 \pm 1.6 \mathrm{~nm}$ and $35.6 \pm 2.1 \mathrm{~nm}$, respectively (Fig. 1C). The in vitro drug release profiles of free CLT and K3-HBc/CLT NCs were performed in phosphate buffer at $\mathrm{pH}$ 7.4. In Fig. 1D, the release rate of $\mathrm{K} 3-\mathrm{HBc} / \mathrm{CLT}$ exhibited a typical sustaining pattern, much slower than that of free CLT, suggesting that $\mathrm{K} 3-\mathrm{HBc} / \mathrm{CLT} \mathrm{NCs}$ remained stable at physiological $\mathrm{pH}$ to avoid premature release.

\section{In vitro intracellular uptake of $\mathrm{K3}-\mathrm{HBC} / \mathrm{CLT}$ NCs}

To evaluate the cellular internalization process, Cy5.5labeled K3-HBc/CLT and HBc-183/CLT NCs were incubated with human proximal tubular epithelial cells (HK-2) cells. As shown by confocal laser scanning microscope images, K3-HBc/CLT NCs were more effectively uptake by HK- 2 cells as compared to HBc-183/CLT NCs at 0.5 and $1 \mathrm{~h}$ (Fig. 2A, B). The fluorescence signals were also detected using flow cytometry to accurately quantify HBc-183/CLT or K3-HBc/CLT NCs uptake at 0.5, 1, 2 and $4 \mathrm{~h}$ (Fig. 2C, D). In Fig. 2E, the median fluorescence intensity values of $\mathrm{K} 3-\mathrm{HBC} / \mathrm{CLT}$ treated cells were significantly higher than that of HBc-183/CLT-treated cells at the indicated time points and exhibited a time dependent increase of fluorescence intensity. These findings suggested that K3-HBc/CLT NCs were more efficiently uptake by HK-2 cells. Considering that endocytic receptor megalin is abundantly expressed in renal tubular epithelial cells [23], it is reasonable for $\mathrm{K} 3-\mathrm{HBc}$ to present the high-efficiency endocytosis. Since previous report showed that K3 peptide binds megalin receptor with high affinity [17], it is necessary to confirm whether K3-HBc 

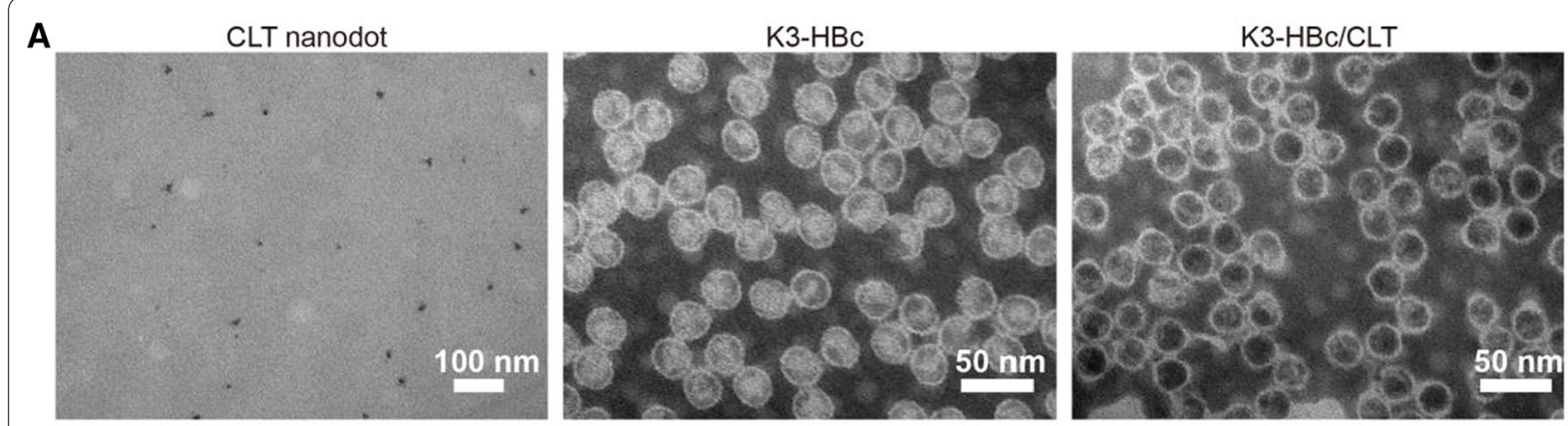

B

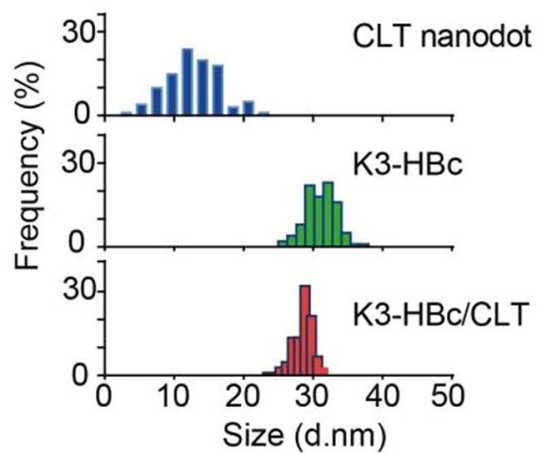

C

$-\mathrm{K} 3-\mathrm{HBc} / \mathrm{CLT}$ $-\mathrm{K} 3-\mathrm{HBC}$

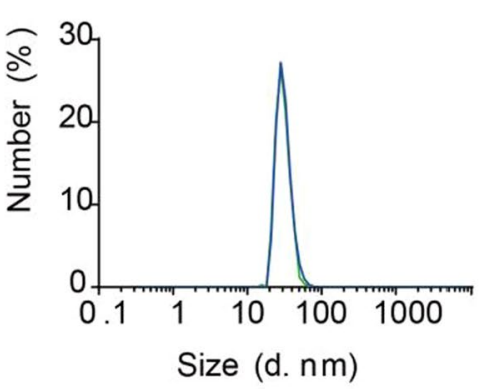

D

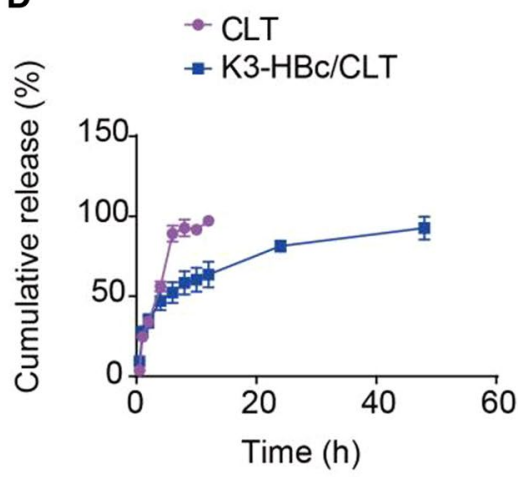

Fig. 1 Characterization of K3-HBC/CLT NCs. A TEM images of CLT nanodots, K3-HBC NCs and K3-HBC/CLT NCs. B Histogram analysis of CLT nanodots, $\mathrm{K} 3-\mathrm{HBC}$ NCs and K3-HBC/CLT NCs, respectively. C The hydrodynamic diameters of K3-HBC and K3-HBC/CLT NCs. D Cumulative release profiles of free $\mathrm{CLT}$ and $\mathrm{K} 3-\mathrm{HBC} / \mathrm{CLT}(\mathrm{n}=3)$

NCs can effectively bind to HK-2 cells via the specific recognition of megalin receptors. We tested the specificity of K3-HBc/CLT NCs for megalin using siRNA-mediated knockdown (Gene ID: LRP2) in HK-2 cells. Megalin protein status was evaluated $72 \mathrm{~h}$ post-siRNA treatment by western blot. The megalin expression level was generally reduced to $59.3 \pm 11.9 \%$ of control levels estimated (Fig. 3A, B). Remarkably, knockdown of megalin expression in HK-2 cells resulted in $58.4 \pm 2.1 \%$ suppression of K3-HBc/CLT uptake (Fig. 3C, D).These findings demonstrated that $\mathrm{K} 3-\mathrm{HBc} / \mathrm{CLT}$ NCs could be internalized efficiently through the specific interaction between $\mathrm{K} 3$ peptide and megalin receptor.

\section{Biodistribution of K3-HBc/CLT NCs}

Next, we investigated the biodistribution of $\mathrm{K} 3-\mathrm{HBc} /$ CLT NCs in vivo. The mice on day 1 or 7 after the UUO were injected with the Cy5.5-labeled K3-HBc/CLT and HBc-183/CLT NCs, respectively. Four hours post injection, the major organs were harvested, and subsequently observed via a near-infrared fluorescent imaging system. The ex vivo biodistribution studies showed significantly different organ distribution patterns for $\mathrm{K} 3-\mathrm{HBc} /$ CLT and HBc-183/CLT NCs (Fig. 4A, B). HBc-183/CLT
NCs exhibited prominent uptake in the liver. Meanwhile, the fibrotic kidney uptake was significantly higher in K3-HBc/CLT group than that in HBc-183/CLT group on day 1 or 7 after the UUO (Fig. 4C, D). Obviously, UUO resulted in the destruction of the glomerular filtration barrier, and the presence of leakage and abnormal fenestrae allowed K3-HBc/CLT to cross the barrier and effectively target the tubular epithelial cells. Furthermore, K3-HBc/CLT or HBc-183/CLT NCs were conjugated with fluorescein isothiocyanate (FITC) to identify the targeted cells. Immunohistochemistry examination of kidney showed that FITC-labeled K3-HBc NCs was exclusively found in the renal cortex (Fig. 4E). Higher magnification revealed a specific uptake at the apical side of tubule cells, where the expression of megalin was abundant [24]. This result further revealed that K3-HBc NCs could effectively recognize tubule cells without a loss in specificity.

\section{Improved efficacy of K3-HBC/CLT NCs in UUO-induced renal fibrosis}

To examine the therapeutic efficacy of K3-HBc/CLT $\mathrm{NCs}$, we investigated their ability to reduce extensive accumulation of extra-cellular matrix within the 


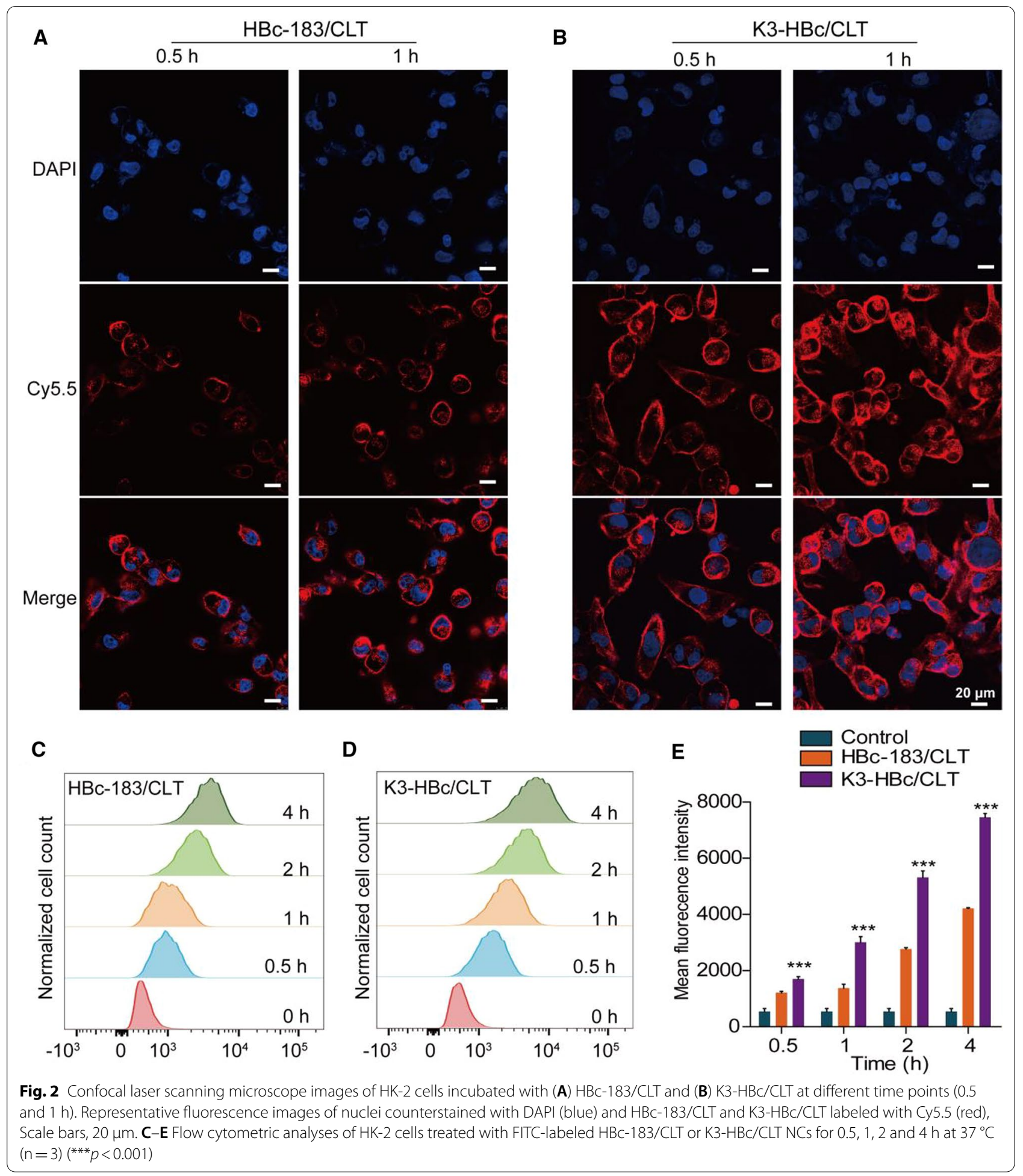

interstitium in UUO-induced renal fibrosis. We first confirmed that $\mathrm{K} 3-\mathrm{HBc}$ NCs could not prevent UUOinduced renal fibrosis (Additional file 1: Figure S5). The induction of renal fibrosis and treatment in mice were schematically presented in Fig. 5A. The renal protective effect of $\mathrm{K} 3-\mathrm{HBc} / \mathrm{CLT}$ NCs was observed by the morphology of kidney in each group (Fig. 5B). The major characteristics observed in obstructed kidneys of mice 


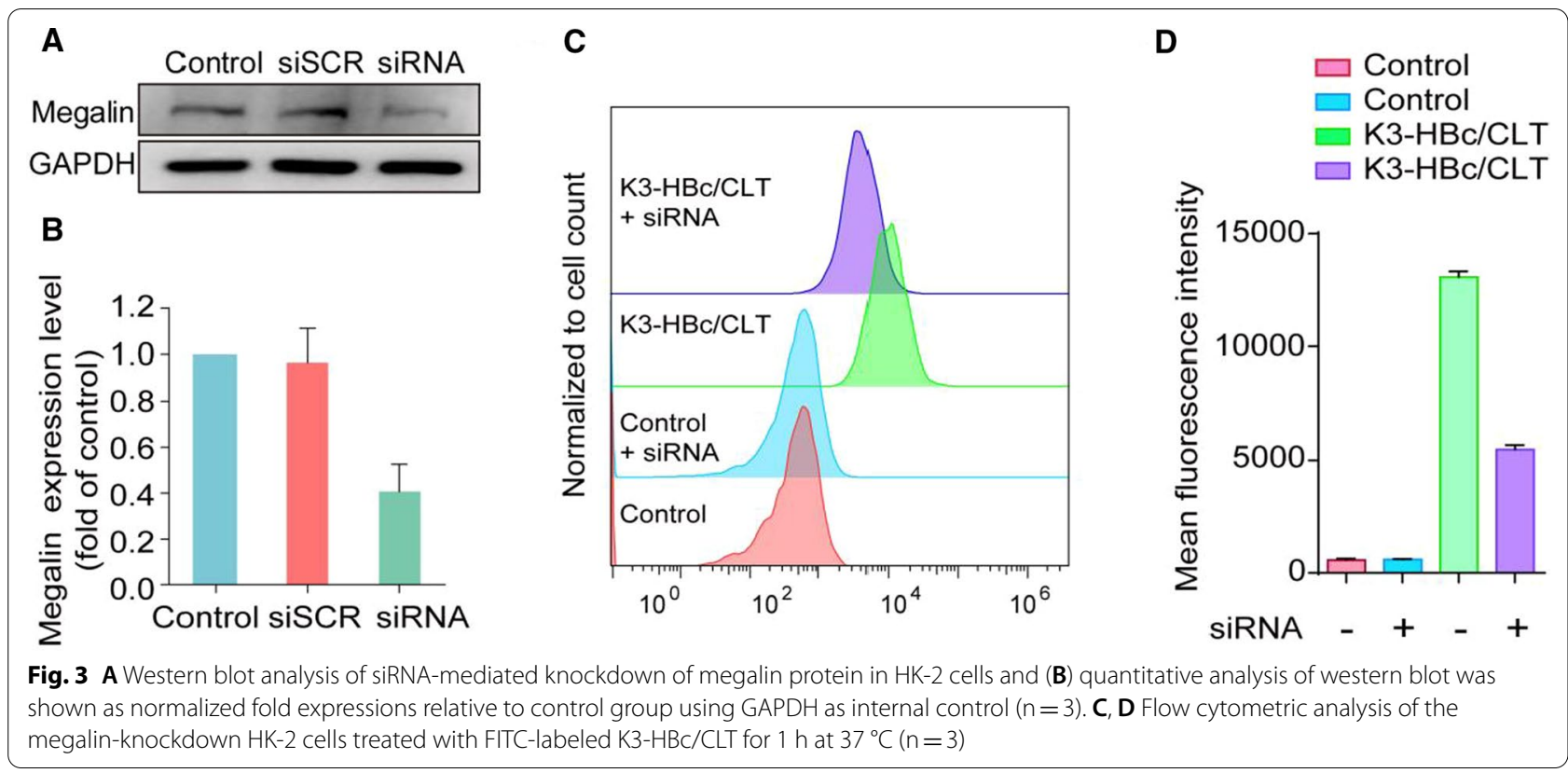

were fluid retention and swollen. Moreover, the left kidney index $(1.26 \pm 0.12 \%)$ in $\mathrm{UUO}+\mathrm{K} 3-\mathrm{HBc} / \mathrm{CLT}$ group was remarkably lower than that in $\mathrm{UUO}+0.9 \% \mathrm{NaCl}$ group $(3.10 \pm 0.39 \%)(p<0.01)$ or in $\mathrm{UUO}+\mathrm{CLT}$ group $(p<0.5)$ (Fig. 5C). Obviously, K3-HBc/CLT appeared to prevent obstructed kidney enlargement more effectively than free CLT.

To evaluate the impact of K3-HBc/CLT NCs on TGF- $\beta$ induced EMT processes in vitro, we measured the expression levels of E-cadherin, fibronectin and vimentin in TGF- $\beta$-induced HK-2 cells (Additional file 1: Figure S6). As expected, the E-cadherin expression was decreased, while the fibronectin and vimentin expression level were increased relative to those in control cells. However, cells treated with TGF- $\beta+$ CLT or TGF- $\beta+$ K3-HBc/CLT NCs exhibited restoration of those pro-fibrotic markers, indicating that CLT and $\mathrm{K} 3-\mathrm{HBc} / \mathrm{CLT}$ NCs could prevent the TGF- $\beta$-induced EMT processes.

The pathological and molecular changes of kidney in different groups were further evaluated. Histological investigation revealed that marked tubular dilation and atrophy, interstitial matrix deposition caused by UUO were greatly ameliorated by K3-HBc/CLT NCs (Fig. 5D). Remarkably, interstitial collagen deposition in obstructed kidney was significantly reduced by $\mathrm{K} 3-\mathrm{HBc} /$ CLT NCs (Fig. 5E). Likewise, the fibrotic area of kidney $(92.90 \pm 9.02)$ in UUO $+\mathrm{K} 3-\mathrm{HBc} / \mathrm{CLT}$ group was further less than that $(131.40 \pm 9.19)$ in UUO + CLT group (Fig. 5G). The immunostaining of fibrotic markers in kidney revealed that K3-HBc/CLT-treated mice exhibited a more significant reduction in obstruction-induced
$\alpha$-SMA, TGF- $\beta$ expressions or collagen I accumulation, compared with CLT-treated mice (Fig. 5F, H-J). Similar results were observed in the western blot studies (Fig. $5 \mathrm{~K}$ ). The expressions of $\alpha$-SMA and vimentin in obstructed kidney were all significantly downregulated in $\mathrm{UUO}+\mathrm{K} 3-\mathrm{HBc} / \mathrm{CLT}$ group (Fig. 5L, M). Taken together, these results demonstrated that K3-HBc/CLT NCs exhibited a better anti-fibrotic effect than CLT on renal fibrosis.

We investigated the systemic toxicity of CLT or $\mathrm{K} 3-\mathrm{HBc} / \mathrm{CLT} \mathrm{NCs}$ in mice on day 14 after treatment. We noticed that mice underwent a steady body weight loss during treatment with free CLT (Fig. 5N). On the contrary, the $\mathrm{K} 3-\mathrm{HBc} / \mathrm{CLT}$-treated mice gained weight similar to the mice in sham group. K3-HBc/CLT NCs displayed an advantage over free CLT concerning the body weight loss. $\mathrm{H} \& \mathrm{E}$ staining revealed that $\mathrm{K} 3-\mathrm{HBc} / \mathrm{CLT}$ NCs effectively prevented serious heart, liver, and brain damage caused by free CLT. No obvious toxic effects were observed in the spleen and lung in $\mathrm{UUO}+\mathrm{K} 3-\mathrm{HBc} / \mathrm{CLT}$ group (Additional file 1: Figure S7). Moreover, K3-HBc/ CLT NCs significantly decreased the elevated blood levels of aminotransferase (AST), aminotransferase (ALT), lactate dehydrogenase (LDH-L), and creatinine (CREA) caused by UUO, but had no significant differences in blood urea nitrogen (BUN) and total bilirubin (TBiL) levels (Additional file 1: Figure S8). In detail, $\mathrm{K} 3-\mathrm{HBc} /$ CLT NCs could decrease the ALT level from $77.25 \pm 1.01$ $\mathrm{U} / \mathrm{L}$ in $\mathrm{UUO}+0.9 \% \mathrm{NaCl}$ group to $52.84 \pm 1.40 \mathrm{U} / \mathrm{L}$ $(p<0.001)$ as well as the AST level from $112.82 \pm 8.86 \mathrm{U} / \mathrm{L}$ in $\mathrm{UUO}+0.9 \% \mathrm{NaCl}$ group to $69.66 \pm 3.78 \mathrm{U} / \mathrm{L}(p<0.01)$. 


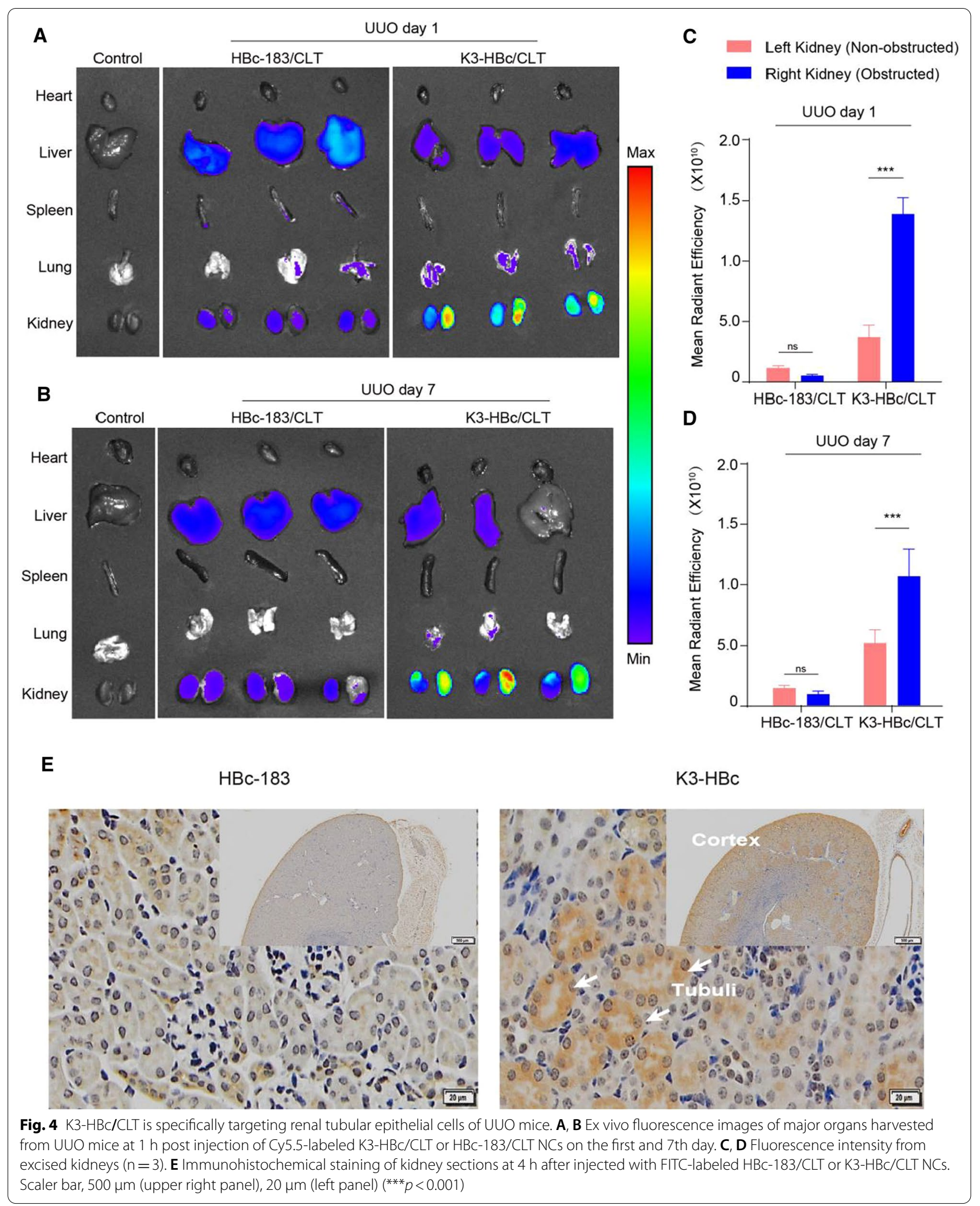


(See figure on next page.)

Fig. 5 Anti-renal fibrosis effects of $\mathrm{K} 3-\mathrm{HBC} / \mathrm{CLT}$ in UUO-induced renal fibrosis. A Flow diagram of the treatment of $0.9 \% \mathrm{NaCl}, \mathrm{CLT}$ or $\mathrm{K} 3-\mathrm{HBC} /$ CLT against the UUO mice model. B The gross-morphological images of kidney tissue from each group. C Kidney index of the UUO mice were measured (obstructed kidney weight/body weight $\times 100$ or non-obstructed kidney/body weight $\times 100)(n=5)$. D Representative images of H\&E staining of kidney sections after different treatments as indicated. E Representative images of Masson's trichrome staining of kidney sections after different treatments as indicated. G Quantification of Masson's trichrome positive area of collagen-like matrix deposition $(n=6)$. F Representative micrographs of the expression and distribution of a-SMA, TGF- $\beta$ and collagen I in kidney tissues sections using immunohistochemical staining and the semi-quantitative IOD analyses of $(\mathbf{H})$ a-SMA, (I) TGF- $\beta$ and $(\mathbf{J})$ collagen I $(n=6)$. K Western blot analyses of a-SMA and Vimentin in mice on day 14 after treatment with CLT or K3-HBC/CLT and quantitative analyses of protein levels of $(\mathbf{L})$ a-SMA and $(\mathbf{M})$ Vimentin $(n=3)$. $\mathbf{N}$ The body weights within 2 weeks measured every two days in different groups $(n=5)$. Scale bar, $100 \mu \mathrm{m}\left({ }^{*} p<0.05,{ }^{* *} p<0.01,{ }^{* * *} p<0.001\right)$

The elevation of CREA in $\mathrm{UUO}+0.9 \% \mathrm{NaCl}$ group $(24.91 \pm 0.67 \mathrm{mmol} / \mathrm{L})$ could be decreased by K3-HBc/ CLT $(18.45 \pm 1.65 \mathrm{mmol} / \mathrm{L})(p<0.05)$. The serum level of LDH-L was $335.56 \pm 21.68 \mathrm{U} / \mathrm{L}$ in $\mathrm{UUO}+\mathrm{K} 3-\mathrm{HBc} /$ CLT group, which was significantly lower than that in $\mathrm{UUO}+0.9 \% \mathrm{NaCl}$ group $(458.57 \pm 8.77 \mathrm{U} / \mathrm{L})(p<0.01)$. We also evaluated the systematic toxicity of K3-HBc NCs in vivo. As shown in Additional file 1: Figure S9, K3-HBCtreated mice maintained the level of ALT and AST within the normal range. Besides, the H\&E staining of sliced organs showed no sign of early lesions in $\mathrm{K} 3-\mathrm{HBc}$ group. The profile of cytokines such as Tumour necrosis factor $\alpha$ (TNF- $\alpha$ ), Interleukin-12 (IL-12), or Interleukin-6 (IL-6) in the serum of healthy mice treated with K3-HBc NCs was analyzed. There was no significant difference between the treated and the control groups (Additional file 1: Figure S10). These results indicated that $\mathrm{K} 3-\mathrm{HBc} \mathrm{NCs}$ could serve as a safe carrier. Taken together, K3-HBc/CLT NCs could improve the CLT therapeutic effect without severe toxic effect.

\section{K3-HBc/CLT NCs exerts marked anti-fibrotic effect through inhibiting cell senescence in UUO mice}

Previous report indicated that the cell senescence of tubular epithelial cells contributed to renal fibrosis [25, 26]. Meanwhile, stable cell cycle arrest, a defining feature of senescence [27], is a functional consequence of EMT program during fibrotic injury [28]. To better understand the anti-fibrotic role of cell scenecence in response to $\mathrm{K} 3-\mathrm{HBc} / \mathrm{CLT} \mathrm{NCs}$, high throughput RNA-seq was performed in each group. We compared biological processes corresponding with cell senescence including DNA damage response, cell cycle arrest and cell cycle checkpoint in all three comparisons by gene ontology (GO) enrichment analysis (Fig. 6A). In addition, persistent DNA damage response (DDR) due to chronic genomic stress or telomere attrition leads to activation of $\mathrm{p} 53$, which in turn activates $\mathrm{p} 21^{\mathrm{Cip} 1}$ to initiate cell cycle arrest $[29,30]$. The core signaling pathways were related with p53, cell cycle and senescence (Fig. 6B). By Kyoto Encyclopedia of Genes and Genomes (KEGG) enrichment analysis, the heatmap of regulated genes expression related to $\mathrm{p} 53$, cell cycle and senescence in each group was shown in Fig. 6C. 11 individual genes in these categories, including Cdknla, Ccne1, Ccne2, Cdk4, Ccnd1, Ccnd2, Sfn, Rprm, Ccnb1, Ccnb2, Cdk1 and GADD45, were significantly regulated in $\mathrm{UUO}+0.9 \% \mathrm{NaCl}$ group compared to sham group. The changes in expression levels for GADD45, Sfn, and Cdk4 genes revealed by quantitative real-time PCR (qPCR) were similar to those determined by the RNASeq analysis (Additional file 1: Figure S11). K3-HBc/CLT NCs largely reversed the alterations in expression levels of the genes caused by UUO. Therefore, it was reasonable to speculate that these cell senescence and cell cycle genes acted vigorously in K3-HBc/CLT NCs mediated anti-fibrotic effect.

To solidify this conclusion, we examined the effect of CLT and K3-HBc/CLT NCs on cell senescence. Increased $\beta$-galactosidase activity and elevated transcript or protein levels of $\mathrm{p} 16^{\text {Ink4a }}$ and $\mathrm{p} 21^{\text {Cip1 }}$ are key molecular characteristics of senescent cells [31]. In vitro senescent HK-2 cells induced by Ang-II were incubated with CLT or $\mathrm{K} 3-\mathrm{HBc} / \mathrm{CLT} \mathrm{NCs}$, following $\beta$-galactosidase staining [32]. Cell senescence in UUO kidkey as indicated by SA- $\beta$-Gal staining was decreased by CLT and K3-HBc/ CLT NCs (Fig. 6D, E). Next, we examined the expression of cell senescence marker by immunocytochemistry. The expression of $\mathrm{p} 21^{\mathrm{Cip} 1}$ and $\mathrm{p} 16^{\mathrm{Ink} 4 \mathrm{a}}$ were remarkably elevated in $\mathrm{UUO}+0.9 \% \mathrm{NaCl}$ group, but $\mathrm{K} 3-\mathrm{HBc} / \mathrm{CLT} \mathrm{NCs}$ and CLT could significantly downregulate these expressions in obstructed kidney (Fig. 6F). Semi-quantitative analyses showed that K3-HBc/CLT NCs could downregulate expression of $\mathrm{p} 16^{\mathrm{Ink} 4 \mathrm{a}}$ and $\mathrm{p} 21^{\mathrm{Cip} 1}$ in obstructed kidney nobly, and $\mathrm{K} 3-\mathrm{HBc} / \mathrm{CLT}$-treated mice expressed p21 ${ }^{\text {Cip } 1}$ protein at lower level compared with CLT-treated mice (Fig. 6G, H). Based on these results, we concluded that K3-HBc/CLT NCs exerted protective effect against renal fibrosis through ameliorating cell senescence.

\section{Discussion}

Regardless of the underlying initiating cause, renal fibrosis leads to loss of function and end-stage renal failure [33], requiring all-life dialysis or kidney transplantation. 


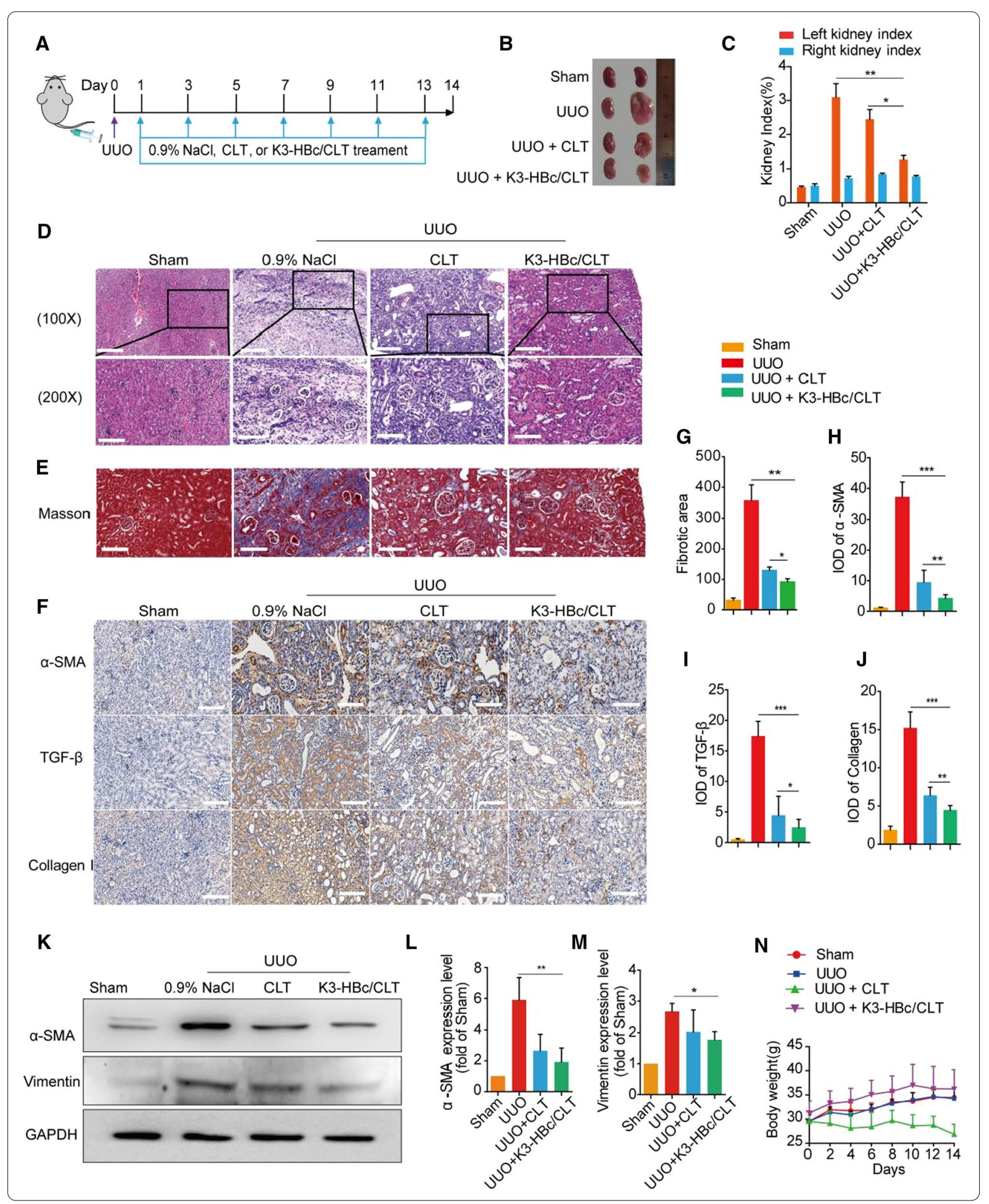




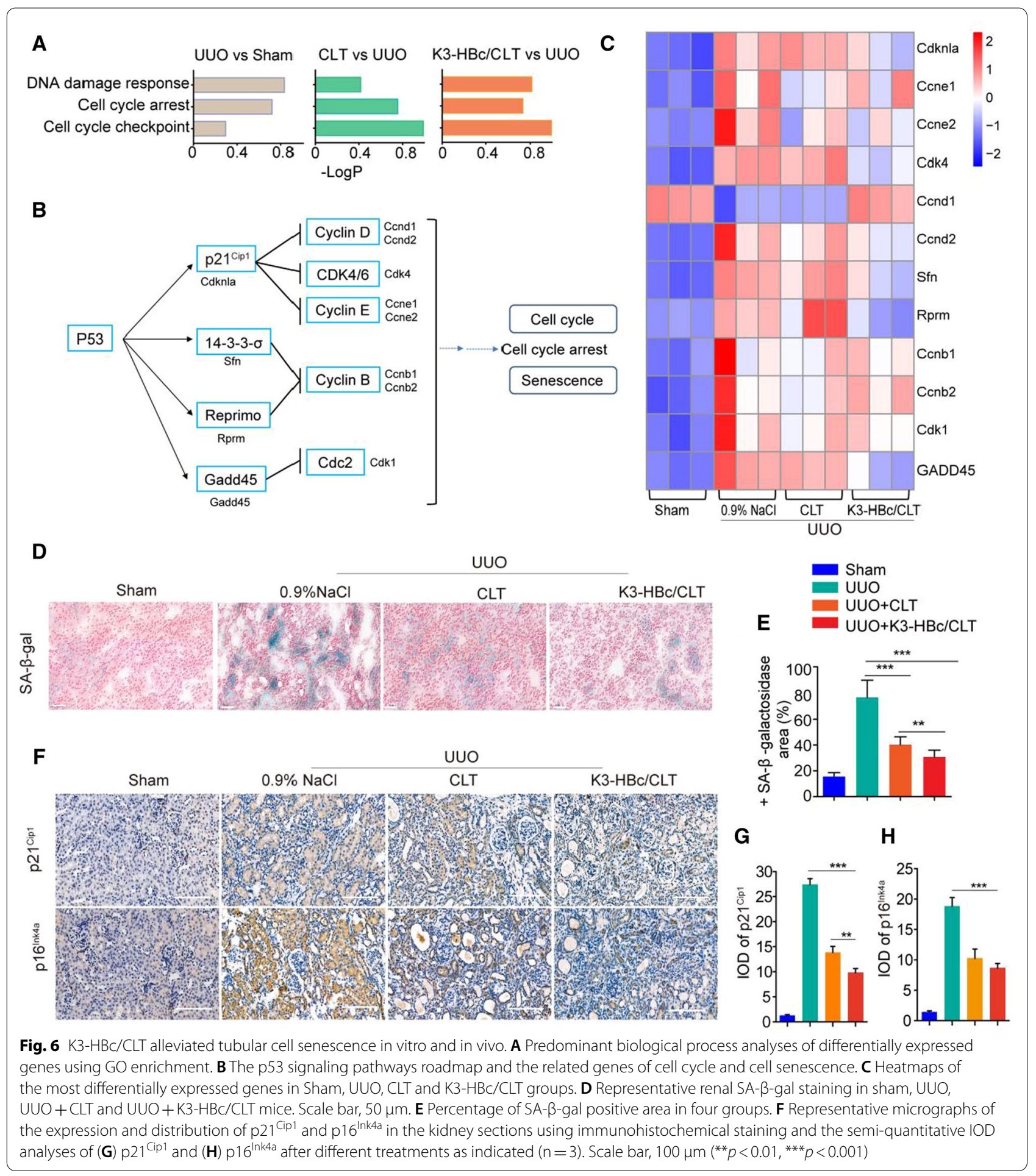

Considering the difficulty of dealing with the underlying process, a specific and effective anti-fibrotic therapy is urgently needed. Here, we found that CLT showed potent protection of kidney function and effects against renal fibrosis, consistant with previous report [34].
Targeted delivery of CLT to tubular epithelial cells using $\mathrm{K} 3-\mathrm{HBc}$ NCs has demonstrated to circumvent restraints such as systemic toxic effects. Li et al. reported that targeted loading of CLT by CREKA-coupled liposomes to interstitial myofibroblast allowed the drug to alleviate 
UUO-induced renal fibrosis [35]. Meanwhile, targeted delivery of celastrol to mesangial cells is also effective against mesangio proliferative glomerulonephritis [12]. Comparing to these findings, $\mathrm{K} 3-\mathrm{HBc} / \mathrm{CLT} \mathrm{NCs}$ in our study were expected to see further improvement in the efficacy for renal fibrosis, since tubular epithelial cells could synthesize and secrete varieties of bioactive molecules that drive interstitial inflammation and fibrosis $[8$, $36,37]$.

Precise delivery of therapeutic agent to tubular epithelial cells by $\mathrm{K} 3-\mathrm{HBc} \mathrm{NCs}$ to prevent maladaptive repair would be a promising strategy for halting the fibrotic process [38]. The main features of K3-HBc NCs include: (i) superb biocompatibility and biodegradability; (ii) well-defined biological structure; (iii) tailored modifications by synthetic biology strategy; (iv) capability of selfassembly and a large internal cavity; (v) suitability for imparting non-natural functions by chemical functionalization. K3-HBc/CLT NCs made the special target-cell contact by binding to megalin via the K3 peptide [17]. In addition, megalin possesses an intracellular domain with a signaling domain for clathrin-mediated endocytosis and allows for kidney-specific delivery in fibrosis [39].As a result, K3-HBc/CLT NCs binding efficiently triggered tubular epithelial cells uptake through receptor-mediated endocytosis [40]. Our results demonstrated K3-HBc/CLT NCs could precisely mimic the structure of the authentic virus, specifically interacted with tubular epithelial cells in a virus-like manner.

The design of $\mathrm{K} 3-\mathrm{HBc}$ NCs is a valuable strategy to enhance CLT specificity for therapeutic applications. Targeted delivery of CLT by K3-HBc/CLT NCs to tubular epithelial cells significantly alleviated collagen deposition and myofibroblast infiltration in UUO-induced renal fibrosis. Moreover, K3-HBc/CLT NCs improved the pharmacokinetics and bioavailability of CLT with reduced drug accumulation in non-targeted tissue and organs, thereby minimizing CLT-related toxicity [41]. Importantly, future clinical application of CLT seems to be limited by its poor water solubility [42]. With K3-HBc NCs, CLT could been given by intravenous injection, making substantial progress in the future clinical application of CLT. In addition, many kidney-targeted drug delivery systems employ synthetic carriers, such as limposomes and micells, have been extensively investigated $[43,44]$. However the high cost, low purity, and variations remain the major obstacles to clinical translation. The well-defined K3-HBc NC can be produced with high yields in $E$. coli expression system without any additional chemical modification, which makes it easy to scale up and may be more conducive to clinical transformation.

Transcriptome sequencing by RNA-seq provided novel perspectives to better understand the molecular mechanisms on attenuation of renal fibrosis by $\mathrm{K} 3-\mathrm{HBc} /$ CLT NCs. In particular, we were able to find strong upregulation of cell senescence and cell cycle genes in UUO-injured kidney. Previous studies have suggested that the structural and functional alterations caused by cellular senescence in tubule cells may play an essential role in the initiation and/or progression of renal fibrosis [45]. The transcriptome analyses showed that there was a close lineage relationship between cell senescence and UUO-injured kidney. Senescence can produce various pro-fibrotic and pro-inflammatory cytokines to promote renal fibrosis [8]. Pharmaceutical blockade of these effects or elimination of senescent cells can counteract fibrosis [46, 47]. In our study, K3-HBc/CLT NCs as well as CLT effectively prevented the UUO-induced up-regulation of cell senescence signaling. Hence, tubule-specific K3-HBc/CLT NCs extraordinarily prevented an early event that occurred before the onset of renal fibrosis via inhibition of cell senescence.

\section{Conclusions}

In summary, we designed an intriguing new method to deliver CLT specifically to tubular epithelial cells by $\mathrm{K} 3-\mathrm{HBc}$ NCs via binding to the megalin receptor. $\mathrm{K} 3-\mathrm{HBC} / \mathrm{CLT}$ NCs significantly alleviated renal fibrosis without severe systemic toxicity. Importantly, $\mathrm{p}^{2} 1^{\mathrm{Cip} 1}$ and p16 ${ }^{\text {Ink4a }}$ pathway was found to be the novel anti-fibrotic molecular basis of K3-HBc/CLT NCs to prevent UUOinduced premature senescence in tubular epithelial cells. The targeted delivery of anti-fibrotic drugs by using K3-HBc/CLT NCs provided an effective and promising therapeutic strategy for renal fibrosis.

\section{Methods \\ Materials}

Celastrol (98.0\%) was purchased from Sichuan Weikeqi Biotech Co., Ltd. FITC and Cy5.5-NHS were purchased from MedChemExpress. Pluronic F-127 was purchased from Sigma-Aldrich. All the chemicals used in this study were of analytical grade.

\section{Preparation of K3-HBc/CLT NCs}

The amino acid sequence of $\mathrm{HBC}-183$ is the same as the previously reported [48]. K3 peptide accompanied with two glycine-rich linkers was incorporated to the surfaceexposed major immunodominant loop region of $\mathrm{HBc}$ 183 (between the residues 78 and 81) to yield K3-HBc $\mathrm{NCs}$. The objective plasmid carrying the $\mathrm{K} 3-\mathrm{HBc}$ gene was purchased from Shanghai Generay Biotech Co., Ltd. The corresponding vectors were transformed into $E$. coli strain BL21 (DE3). The expression and purification of $\mathrm{K} 3-\mathrm{HBc}$ protein were performed as the previous description [49]. 
Ultra-small CLT nanodots were fabricated by dropletconfined crystallization during the freeze-drying of frozen emulsion [22]. CLT-containing dichloromethane was mixed with an aqueous solution containing F127 (1.0 wt\%), and then converted into an $\mathrm{O} / \mathrm{W}$ emulsion by sonification. The emulsion was immediately immersed in liquid nitrogen and rapidly frozen, followed by freezedrying. The resulted CLT nanodots were harvested and washed by centrifugation-redispersion cycles to remove free F127.

The bioengineered $\mathrm{K} 3-\mathrm{HBc}$ NCs were reversibly disassembled with $8 \mathrm{M}$ urea at $25{ }^{\circ} \mathrm{C}$ for $3 \mathrm{~h}$. After the dissociation, ultra-small CLT nanodots were added and the solution was gently stirred for $2 \mathrm{~h}$. Then, the reassembly of K3-HBc NCs was conducted by dialysis in an assembling buffer at $4{ }^{\circ} \mathrm{C}$ and free CLT nanodots was removed by SuperdexTM-75 column. To calculate the loading capacity, the concentration of CLT was determined by UV-vis spectrophotometer.

The morphology of ultra-small CLT nanodots, K3-HBc $\mathrm{NCs}$ and $\mathrm{K} 3-\mathrm{HBc} / \mathrm{CLT} \mathrm{NCs}$ were observed using a transmission electron microscope (TEM, JEM-1400, JEOL). The hydrodynamic diameter sizes of NCs and zeta potential of CLT nanodots were respectively determined by Zeta Sizer (Nano Series, Malvern). Besides, the diameter distributions of CLT nanodots and NCs were obtained through counting at least 100 particles in TEM images.

The FITC-labeled HBc-183 or K3-HBc NCs were performed as the previous description [50]. Briefly, FITC was dissolved in DMSO, and then were mixed with $\mathrm{HBc}-$ 183 or K3-HBc NCs and gently vortexed for $2 \mathrm{~h}$ in the dark at $25{ }^{\circ} \mathrm{C}$. The mixtures were dialyzed in PBS buffer at $4{ }^{\circ} \mathrm{C}$ for $48 \mathrm{~h}$ to remove unreacted FITC molecules. Cy5.5-NHS was labeled to $\mathrm{HBc}-183$ or K3-HBc NCs in the same methods with FITC labeling.

\section{In vitro drug release}

Dialysis bags $(\mathrm{MWCO}=3000 \mathrm{Da})$ containing $2 \mathrm{~mL}$ of K3-HBc/CLT and CLT solution (CLT, $2 \mathrm{mg}$ ) were separately submerged in release medium $(50 \mathrm{~mL})$ at $37{ }^{\circ} \mathrm{C}$ while stirring at $100 \mathrm{r} / \mathrm{min}$. At predetermined time points $(0.25,0.5,1,2,4,8,12,24,36$ and $48 \mathrm{~h}), 1 \mathrm{~mL}$ aliquots of the solution were withdrawn to determinate the released drug. Then an equal volume of fresh buffer was added to keep the volume constant and to ensure sink conditions throughout the whole process. These samples were determined by high performance liquid chromatography (HPLC) to calculate the cumulative amount of released drug and plot the percentage of drug released.

\section{Animals model and therapeutic experiments}

The male Sprague Dawley rats (6-8 weeks) and male BALB/C mice (6-8 weeks) were purchased from animal center in Third Military Medicine University (Chongqing, China). All animal care and experiments were conducted in compliance with the requirements of the National Act on the use of experimental animals (China) and were approved by the Institutional Animal Care and Ethic Committee of Third Military Medicine University.

UUO was a well-established experimental model of renal fibrosis [51]. Animals were anesthetized with $1 \%$ pentobarbital $\left(10 \mu \mathrm{L} \mathrm{g}^{-1}\right)$, the left ureter was exposed by a tilted incision which near the lower edge of the left rib $1 \mathrm{~cm}$, and then it was obstructed by two-point ligations with 4-0 silk sutures. The abdominal incision was sealed with the same silk suture, and animals were returned to the cages. Sham-operated mouse underwent the same procedure except ligation.

The rats were randomized into three groups: the sham group treated with PBS, the UUO group treated with $0.9 \% \mathrm{NaCl}$, and UUO + CLT group received free CLT $\left(1 \mathrm{mg} \mathrm{kg}^{-1}\right)$. PBS, $0.9 \% \mathrm{NaCl}$ or free CLT was injected intraperitoneally every two days. The body weights were recorded every two days. All animals were sacrificed on day 14. Kidney index from each group were measured (obstructed kidney weight/body weight $\times 100 \%$ or nonobstructed kidney/body weight $\times 100 \%$ ). The heart, liver, spleen, lung kidney and brain were harvested immediately. Tissues were washed, fixed and stained with $H \& E$. Histological lesions were observed under the microscope (Olympus BX51, Japan).

To study the therapeutic effect of K3-HBc/CLT in the UUO model, 24 mice were randomly divided into four groups $(n=6)$ : sham, $\mathrm{UUO}+0.9 \% \mathrm{NaCl}, \mathrm{UUO}+\mathrm{CLT}$ and $\mathrm{UUO}+\mathrm{K} 3-\mathrm{HBc} / \mathrm{CLT}$ groups. The mice were treated with CLT $\left(1 \mathrm{mg} \mathrm{kg}^{-1}\right)$ by intraperitoneal injection or K3-HBc/CLT (CLT, $1 \mathrm{mg} \mathrm{kg}^{-1}$ ) by tail vein injection. Both CLT and K3-HBc/CLT were administered every two days. The mice were treated with an equal volume of $0.9 \% \mathrm{NaCl}$ in $\mathrm{UUO}+0.9 \% \mathrm{NaCl}$ group. All mice were sacrificed on day 14. The organs were harvested for $\mathrm{H} \& \mathrm{E}$ staining. Before animals were sacrificed, blood was collected and centrifuged at $2500 \mathrm{rpm}$ for $15 \mathrm{~min}$. The serum was measured on a chambray 240 automatic biochemical analyzer (Radyto, China) for the following analytes: BUN,CREA, AST, ALT, TBIL and LDH-L, which indicates renal, liver and heart functions, respectively.

\section{Cell culture and TGF- $\beta$-induced EMT processes in vitro}

HK-2cells were purchased fromStem Cell Bank, Chinese Academy of Sciences. The HK-2 cells were cultured in Dulbecco's modified Eagle's medium (DMEM)/F12 medium (Hyclone, SH30023.01, Thermo Scientific, USA) supplemented with $10 \%$ fetal bovine serum (ScienCell, Carlsbad, CA, USA), $100 \mathrm{U} \mathrm{mL}^{-1}$ penicillin/streptomycin 
(Beyotime, Shanghai, China). Cells were cultured in a humidified atmosphere containing $5 \% \mathrm{CO}_{2}$ at $37^{\circ} \mathrm{C}$.

Serum-starved HK-2 cells were pre-incubated with or without the CLT (500 nM) or K3-HBc/CLT (CLT, $500 \mathrm{nM}$ ) for $1 \mathrm{~h}$ for the in vitro experiments. Then the cells were stimulated with TGF- $\beta 1\left(5 \mathrm{ng} \mathrm{mL}{ }^{-1}\right)$ at the indicated time points. HK-2 cell morphology was observed by phasecontrast microscopy and the cell lysate was harvested for further study.

\section{Cellular uptake of K3-HBc NCs in vitro}

HK-2 cells were seeded onto sterilized microscope coverslips placed in 6-well plates at a density of $2 \times 10^{5}$ cells/well and incubated in DMEM medium supplemented with $10 \%$ fetal bovine serum for $24 \mathrm{~h}$ at $37^{\circ} \mathrm{C}$ with $5 \% \mathrm{CO}_{2}$. Afterwards, the cells were treated with Cy5.5-labeled K3-HBc/ CLT NCs or HBc-183/CLT NCs under the equivalent NCs concentration $\left(20 \mu \mathrm{g} \mathrm{mL}^{-1}\right)$ and co-incubated for 0.5 and $1 \mathrm{~h}$. Subsequently, the cells were washed $5 \mathrm{~min}$ with PBS for three times, fixed with cold $4 \%$ paraformaldehyde for $20 \mathrm{~min}$ and washed three times with PBS. DAPI was used to stain the nuclei of the cells. Finally, the cells were observed with a confocal laser scanning microscope (Zeiss LSM 700, Zeiss, Thornwood, Germany).

The cellular uptake of FITC-labeled-K3-HBc/CLT NCs or HBc-183/CLT NCs in HK-2 cells was quantified by flow cytometry. Briefly, HK- 2 cells $\left(1 \times 10^{5}\right.$ cells/well $)$ were seeded in 12-well plates and incubated for $24 \mathrm{~h}$. Then, cells were incubated with FITC-labeled K3-HBc/ CLT NCs or HBc-183/CLT NCs under the equivalent concentration $\left(20 \mu \mathrm{g} \mathrm{mL}^{-1}\right)$. At predetermined times $(0.5$, 1,2 and $4 \mathrm{~h}$ ), cells were washed with PBS and detached using trypsin. Finally, cells were suspended in cold PBS, which was then immediately analyzed by BD FACS Verse flow cytometer (BD Biosciences, San Jose, CA, USA).

The siRNA transfection experiments were performed as reported previously [52]. The reagents for silencing of gene expression were obtained from Sangon Biotechnology. We employed siRNAs specifically targeting mRNA for humanmegalin and a nontargeting scramblesequence siRNA (siSCR) as a negative control. HK-2 cells were transfected with siSCR or siRNA targeting LRP2 for $72 \mathrm{~h}$. Then cells were incubated with FITC-labeled $\mathrm{K} 3-\mathrm{HBc} / \mathrm{CLT}$ NCs for $1 \mathrm{~h}$, the cellular uptake efficiency was analyzed by flow cytometry.

\section{Distribution of $\mathrm{K} 3-\mathrm{HBc} / \mathrm{CLT}$ NCs in vivo}

Mice on day 1 or day 7 after the UUO received Cy5.5labeled HBc-183/CLT or K3-HBc/CLT NCs via the tail vein. Four hours post injection, the mice were sacrificed and the major organs including heart, liver, spleen, lung and kidney were excised and imaged using an imaging system (IVIS Spectrum, Caliper LifeSciences, USA).

\section{SA- $\beta$-galactosidase staining}

The ability of K3-HBc/CLT to inhibited cell senescence in UUO mice kidney was measured by SA- $\beta-$ Gal staining. Frozen mouse kidney sections ( $4 \mu \mathrm{m}$ thickness) were used for detection of SA- $\beta-G a l$. The activity of SA- $\beta$ Gal was analyzed with SA- $\beta$-Gal staining kit (Beyotime, Shanghai, China) according to the manufacturer's instructions.

\section{Western blot analysis}

Total proteins were isolated from kidney and HK-2 cells and quantified as described previously [53]. Protein samples $(30 \mu \mathrm{g} / \mathrm{lane})$ were resolved using sodium dodecyl sulfate-polyacrylamide electrophoresis and then transferred onto polyvinylidene difluoride membranes (PVDF, Millipore, USA). The membranes were incubated at $4{ }^{\circ} \mathrm{C}$ overnight with $\alpha$-SMA (1:300, Abcam, Cambridge, MA, USA), fibronectin (1:300, Santa Cruz, CA, USA), vimentin (1:500, Santa Cruz, CA, USA), E-cadherin (1:1000, Proteintech, Wuhan, China), Megalin (1:100, Santa Cruz, CA, USA) and HBc (1:1000, Abcam, Cambridge, MA, USA), followed by incubation with horseradish peroxidase-conjugated goat anti-mouse (1:10,000, Santa Cruz, CA, USA) or goat anti-rabbit IgG secondary antibodies (1:10,000, Santa Cruz, CA, USA) at room temperature for $1 \mathrm{~h}$. The immunoblots were imaged by the chemiluminescence western blot detection system (Bio-Rad ChemiDoc MP, California, USA) with GAPDH (1:5000, ZENBIO, Chengdu, China) as the loading control.

\section{qPCR analysis}

Total RNA was extracted from the obstructed kidney mice treated with $0.9 \% \mathrm{NaCl}, \mathrm{CLT}$, or $\mathrm{K} 3-\mathrm{HBc} / \mathrm{CLT}$ for 14 days using Trizol reagent (TaKaRa, Japan) according to the manufacturer's recommendations. Subsequently, $1 \mu \mathrm{g}$ RNA was used to reverse transcribed cDNA by PrimeScript RT reagent Kit with gDNA Eraser (TaKaRa, Japan). cDNA abundance was de-termined by qPCR with SYBRPRIME qPCR Kit (Bioground, China). The following primers were used: Cdknla forward, $5^{\prime}$-tgatctgctgctcttttcc- $3^{\prime}$, and reverse, $5^{\prime}$-tacattcccttccagtcca- $3^{\prime}$; Gadd45 forward, $5^{\prime}$-agaagaccgaaaggatgga- $3^{\prime}$, and reverse, $5^{\prime}$-cgtaatggtgcgctgac- $3^{\prime}$; Sfn forward, $5^{\prime}$-cgacagtgctggggaag- $3^{\prime}$, and reverse, 5'-ccaaggtgtggctgaaca-3'; Rprm forward, $5^{\prime}$-acgcaaacctgtcggagt- $3^{\prime}$, and reverse, $5^{\prime}$-tgccacctgctgctgtat-3'; Cdk4 forward, 5'-gctgaaattggtgtcggt-3', and reverse, $5^{\prime}$-cctccagaatccttaaca-3'; GAPDH forward, $5^{\prime}$-ggttgtctcctgccgacttca-3', and reverse, $5^{\prime}$-tggtccagggtttcttactcc-3'. GAPDH serves as an endogenous control. 


\section{Histology and immunohistochemical staining}

Kidney tissues harvested from animals on day 14 were fixed in $4 \%$ paraformaldehyde, embedded in paraffin and cut into $4 \mu \mathrm{m}$ thick per section. The sections were stained with H\&E and Masson's trichrome staining. The collagen deposition in the obstructed kidney tissue was assessed by Masson's trichrome staining. At least 10 randomly selected cortical fields were observed under the microscope (Olympus BX51, Japan) and the renal fibrotic area was semi-quantitated using image-pro plus 6.0 software.

The immunohistochemistry experiment was performed as previously described [54]. Briefly, sections mounted on slides were blocked with 5\% BSA for $1 \mathrm{~h}$ and incubated over night at $4{ }^{\circ} \mathrm{C}$ with primary antibodies against $\alpha$-SMA (1:200), collagen I (1:500, Santa Cruz, CA, USA), TGF- $\beta$ (1:200), p16 (1:500) and p21 (1:500). The slides were then stained with a goat anti-rabbit IgG secondary antibody (1:50, Beyotime, jiangsu, China). The results were analyzed using a 3,3'-diaminobenzidine (DAB) assay kit (Servicebio, Wuhan, China). The slides were visualized by a microscope (Olympus BX51, Japan) and were semiquantitated based on the intensity and spread of positive staining in terms of IOD using image-pro plus 6.0 software.

To examine the localization of K3-HBc NCs in the kidney, immunohistochemical studies were performed in $\mathrm{BALB} / \mathrm{c}$ mice. The FITC-labeled $\mathrm{K} 3-\mathrm{HBc} \mathrm{NCs}$ or $\mathrm{HBc}-$ $183 \mathrm{NCs}$ was administered to mice via tail vein injection. At $1 \mathrm{~h}$ post injection, mice were sacrificed and harvested kidneys were fixed in $4 \%$ paraformaldehyde for $24 \mathrm{~h}$. The primary antibody was rabbit anti-FITC antibody (1:500, Sangon Biotech, Shanghai, China). The immunohistochemical staining and analysis was performed as described above.

\section{RNA-seq and bioinformatic analysis}

RNA-seq experiment was performed as previous descriped [55]. Briefly, RNA was isolated from kidney tissues in sham, UUO, UUO + CLT and UUO + K3-HBc/ CLT groups using the Trizol kit according to the manufacturer 's instructions, respectively. Sample integrity, quality and purity were determined accordingly. cDNA synthesis was performed from DNase1 treated RNA samples using ImProm-II Reverse Transcriptase (Promega Corp., Madison, WI). Sequencing libraries were generated using NEBNext ${ }^{\circledR}$ UltraTM RNA Library Prep Kit for Illumina ${ }^{\circledR}$ (NEB, USA) following manufacturer's recommendations. The sequencing service is performed by Novogene Corporation (Beijing, China). Raw reads were generated using an Illumina Novaseq 6000 platform by paired-end sequencing. After quality filtering, the clean reads were mapped onto the mouse reference genome and the read count for each gene was derived from the mapping results obtained by Feature Counts. All read counts were normalized to fragments per kilo bases per million mapped reads (FPKM). DEseq2 was used to determine differential expressions. Transcripts with an adjusted p value, 0.05 were accepted as being differential. Differentially expressed genes from the different comparisons and the subjected biological process and molecular functional pathways were analyzed using the Gene Ontology, Kyoto Encyclopedia of Genes and Genomes (KEGG) and Reactome biochemical pathway databases.

\section{Statistical analysis}

Quantitative results were expressed as mean \pm SEM. Statistical differences among groups were checked by One-way ANOVA followed by Newman-Keuls multiple comparisons test or Student's unpaired two-tailed $t$ test from GraphPad Prism 5.0 (GraphPad Software, San Diego, CA, USA). $p<0.05$ was considered statistically significant.

\section{Abbreviations}

ALT: Aminotransferase; a-SMA: a-Smooth muscle actin; AST: Aminotransferase; BUN: Blood urea nitrogen; CKD: Chronic kidney disease; CLT: Celastrol; CREA: Creatinine; EMT: Epithelial-mesenchymal transition; FITC: Fluorescein isothiocyanate; $\mathrm{HBC}$ : Hepatitis B core protein; HK-2: Human proximal tubular epithelial cells; H\&E: Hematoxylin and eosin; IOD: Integrated optical density; LDH-L: Lactate dehydrogenase; NCs: Nanocages; TBIL: Total bilirubin; TEM: Transmission electron microscope; TGF- $\beta$ : Transforming growth factor-beta; TWHF: Tripterygiumwilfordii Hook F; UUO: Unilateral ureteral obstruction; SA- $\beta$-gal: Senescence-associated $\beta$-galactosidase; TNF-a: Tumour necrosis factor a; L-12: Interleukin-12; L-6: Interleukin-6; qPCR: Quantitative real-time PCR.

\section{Supplementary Information}

The online version contains supplementary material available at https://doi. org/10.1186/s12951-021-00900-w.

Additional file 1: Figure S1. CLT suppressed UUO-induced renal fibrosis. Figure S2. Representative H\&E-stained images of heart, liver, spleen, lung and brain on day 14 after treatment. Figure S3. (A) SDS-PAGE analysis of $\mathrm{K} 3-\mathrm{HBC}$ or $\mathrm{HBC}-183$. (B) Western blot analysis of $\mathrm{HBC}-183$ or $\mathrm{K} 3-\mathrm{HBC}$ with antibody against $\mathrm{HBC}$. Figure S4. Zeta potential of ultra-small CLT nanodots. Figure S5. K3-HBC NCs were administered to the mice in UUO $+\mathrm{K} 3-\mathrm{HBC}$ NCs group by tail vein injection at a dosage of $9.49 \mathrm{mg} / \mathrm{kg}$ every other day starting immediately after UUO operation. Figure S6. AntiEMT effects of CLT orK3-HBC/CLT in vitro. Serum-starved HK-2 cells were incubated with CLT or K3-HBC/CLT (500 nM) for $1 \mathrm{~h}$ and then stimulated with TGF- $\beta 1$ (5 ngmL-1) for 48h. Figure S7. Representative H\&E stained images of the organs harvested from the mice after various treatments. Figure S8. Blood biochemistry analyses of the mice after treatment with $\mathrm{CLT}$ or $\mathrm{K3}-\mathrm{HBC} / \mathrm{CLTfor} 14$ days. Figure S9. (A) Blood biochemistry analyses of the healthymice after treatment with $\mathrm{K} 3-\mathrm{HBC}$ for 14 days. The results showed mean and standard deviation of AST, ALT, BUN, CREA, LDH-L, TBiL $(n=3)$. (B) Representative H\&E stained images of the organs harvested from the mice after treatment with $\mathrm{K} 3-\mathrm{HBC}$ for 14 days. Scale bar $=100 \mu \mathrm{m}$. Figure S10. Serum cytokine analysis in mice. Figure S11. mRNA levels of (A) Cdknla, (B) GADD45, (C) Rprm, (D) Sfn, and (E) Cdk4 were measured by qPCR in obstructed kidney from the mice treated with $0.9 \% \mathrm{NaCl}, \mathrm{CLT}$, or K3-HBC/CLT for 14 days $(n=3)$ 


\author{
Acknowledgements \\ Authors are grateful to their respective institutions for support.
}

\section{Authors' contributions}

$X Z, Q C, L Z, H Z, C L Q Y, T L, H Z, X C, L R$, and WS have collected literature and wrote the manuscript. LR, and WS edited the manuscript. All authors read and approved the final manuscript.

\section{Funding}

This work was supported by National Natural Science Foundation of China (31900995, 81901111, U1904206) and the Natural Science Foundation of Chongqing, China (cstc2020jcyj-msxmX0411).

\section{Availability of data and materials}

All data related to the manuscript are available in the manuscript in the form of figures.

\section{Declarations}

\section{Ethics approval and consent to participate}

The study was approved by the Ethics Committee of Third Military Medical University.

\section{Consent for publication}

All authors gave their consent for publication.

\section{Competing interests}

The authors declare no conflict of interest.

\section{Author details}

${ }^{1}$ Department of Pharmacology, College of Pharmacy and Laboratory Medicine, Army Medical University (Third Military Medical University), Chongqing 400038, People's Republic of China. ${ }^{2}$ Biomedical Analysis Center, College of Basic Medicine, Army Medical University (Third Military Medical University), Chongqing 400038, People's Republic of China. ${ }^{3}$ School of Medicine, Xiamen University, Xiamen 361102, People's Republic of China. ${ }^{4}$ School of Life Sciences, Xiamen University, Xiamen 361102, People's Republic of China. ${ }^{5}$ Department of Biomaterials, College of Materials, Xiamen University, Xiamen 361005, People's Republic of China. ${ }^{6}$ School of Medicine, Huaqiao University, Quanzhou 362021, People's Republic of China.

Received: 2 February 2021 Accepted: 18 May 2021

Published online: 26 May 2021

\section{References}

1. Jha V, Garcia-Garcia G, Iseki K, Li Z, Naicker S, Plattner B, Saran R, Wang AY, Yang CW. Chronic kidney disease: global dimension and perspectives. Lancet. 2013;382:260-72.

2. Liu Y. Cellular and molecular mechanisms of renal fibrosis. Nat Rev Nephrol. 2011;7:684-96.

3. Humphreys BD. Mechanisms of renal fibrosis. Annu Rev Physiol. 2018;80:309-26.

4. Klinkhammer BM, Goldschmeding R, Floege J, Boor P. Treatment of renal fibrosis-turning challenges into opportunities. Adv Chronic Kidney Dis. 2017:24:117-29.

5. Sun YBY, Qu X, Caruana G, Li JJD. The origin of renal fibroblasts/ myofibroblasts and the signals that trigger fibrosis. Differentiation. 2016;92:102-7.

6. Gewin LJMB. Renal fibrosis: primacy of the proximal tubule. Matrix Biol. 2018:68-69:248-62

7. Liu B, Tang T, Lv L-L. Biology: how tubular epithelial cell injury contributes to renal fibrosis. Adv Exp Med Biol. 2019;1165:233-52.

8. Allison SJ. Fibrosis: targeting EMT to reverse renal fibrosis. Nat Rev Nephrol. 2015:11:565.

9. Shen B. A new golden age of natural products drug discovery. Cell. 2015:163:1297-300
10. Zhu B, Wang Y, Jardine M, Jun M, Lv JC, Cass A, Liyanage T, Chen HY, Wang YJ, Perkovic V. Tripterygium preparations for the treatment of CKD: a systematic review and meta-analysis. Am J Kidney Dis. 2013;62:515-30.

11. Tang W, Zuo JP. Immunosuppressant discovery from Tripterygium wilfordii Hook f: the novel triptolide analog (5R)-5-hydroxytriptolide (LLDT-8). Acta Pharmacol Sin. 2012;33:1112-8.

12. Guo L, Luo S, Du Z, Zhou M, Li P, Fu Y, Sun X, Huang Y, Zhang Z. Targeted delivery of celastrol to mesangial cells is effective against mesangioproliferative glomerulonephritis. Nat Commun. 2017:8:878.

13. Cascão R, Fonseca JE, Moita LF. Celastrol: A spectrum of treatment opportunities in chronic diseases. 2017:4.

14. Yoo JW, Irvine DJ, Discher DE, Mitragotri S. Bio-inspired, bioengineered and biomimetic drug delivery carriers. Nat Rev Drug Discov. 2011:10:521-35.

15. Hill BD, Zak A, Khera E, Wen F. Engineering virus-like particles for antigen and drug delivery. Curr Protein Pept Sci. 2018;19:112-27.

16. Parodi A, Molinaro R, Sushnitha M, Evangelopoulos M, Martinez JO, Arrighetti N, Corbo C, Tasciotti E. Bio-inspired engineering of cell- and virus-like nanoparticles for drug delivery. Biomaterials. 2017;147:155-68.

17. Wischnjow A, Sarko D, Janzer M, Kaufman C, Beijer B, Brings S, Haberkorn U, Larbig G, Kubelbeck A, Mier W. Renal targeting: peptide-based drug delivery to proximal tubule cells. Bioconjug Chem. 2016;27:1050-7.

18. Ina K, Kitamura H, Tatsukawa S, Fujikura Y. Significance of alpha-SMA in myofibroblasts emerging in renal tubulointerstitial fibrosis. Histol Histopathol. 2011;26:855-66.

19. Rauchman M, Griggs D. Emerging strategies to disrupt the central TGFbeta axis in kidney fibrosis. Transl Res. 2019;209:90-104.

20. Lu Y, Chan W, Ko BY, VanLang CC, Swartz JR. Assessing sequence plasticity of a virus-like nanoparticle by evolution toward a versatile scaffold for vaccines and drug delivery. Proc Natl Acad Sci USA. 2015;112:12360-5.

21. Shan W, Zheng H, Fu G, Liu C, Li Z, Ye Y, Zhao J, Xu D, Sun L, Wang X, et al. Bioengineered nanocage from $\mathrm{HBc}$ protein for combination cancer immunotherapy. Nano Lett. 2019;19:1719-27.

22. Ni DZ, Ding H, Liu S, Yue H, Bao YL, Wang ZH, Su ZG, Wei W, Ma GH. Superior intratumoral penetration of paclitaxel nanodots strengthens tumor restriction and metastasis prevention. Small. 2015;11:2518-26.

23. Tian E, Wang S, Zhang L, Zhang Y, Malicdan MC, Mao Y, Christoffersen C, Tabak LA, Schjoldager KT, Ten Hagen KG. Galnt11 regulates kidney function by glycosylating the endocytosis receptor megalin to modulate ligand binding. Proc Natl Acad Sci USA. 2019:116:25196-202.

24. Smith CP, Lee WK, Haley M, Poulsen SB, Thevenod F, Fenton RA. Proximal tubule transferrin uptake is modulated by cellular iron and mediated by apical membrane megalin-cubilin complex and transferrin receptor 1. J Biol Chem. 2019;294:7025-36.

25. Sturmlechner I, Durik M, Sieben CJ, Baker DJ, van Deursen JM. Cellular senescence in renal ageing and disease. Nat Rev Nephrol. 2017;13:77-89.

26. Jin H, Zhang Y, Ding Q, Wang SS, Rastogi P, Dai DF, Lu D, Purvis M, Cao C, Wang $A$, et al. Epithelial innate immunity mediates tubular cell senescence after kidney injury. JCI Insight 2019;4.

27. Lessard F, Igelmann S, Trahan C, Huot G, Saint-Germain E, Mignacca L, Del Toro N, Lopes-Paciencia S, Le Calve B, Montero M, et al. Senescenceassociated ribosome biogenesis defects contributes to cell cycle arrest through the Rb pathway. Nat Cell Biol. 2018:20·789-99.

28. Lovisa S, LeBleu VS, Tampe B, Sugimoto H, Vadnagara K, Carstens JL, Wu CC, Hagos Y, Burckhardt BC, Pentcheva-Hoang T, et al. Epithelial-to-mesenchymal transition induces cell cycle arrest and parenchymal damage in renal fibrosis. Nat Med. 2015;21:998-1009.

29. Niedernhofer LJ, Gurkar AU, Wang Y, Vijg J, Hoeijmakers JHJ, Robbins PD. Nuclear genomic instability and aging. Annu Rev Biochem. 2018:87:295-322.

30. Reyes J, Chen JY, Stewart-Ornstein J, Karhohs KW, Mock CS, Lahav G. Fluctuations in p53 signaling allow escape from cell-cycle arrest. Mol Cell. 2019;73:1306

31. Childs BG, Durik M, Baker DJ, van Deursen JM. Cellular senescence in aging and age-related disease: from mechanisms to therapy. Nat Med. 2015:21:1424-35

32. Hernandez-Segura A, Nehme J, Demaria M. Hallmarks of cellular senescence. Trends Cell Biol. 2018;28:436-53.

33. Rockey DC, Bell PD, Hill JA. Fibrosis - a common pathway to organ injury and failure. N Engl J Med. 2015;372:1138-49. 
34. Tang M, Cao X, Zhang K, Li Y, Zheng QY, Li GQ, He QH, Li SJ, Xu GL, Zhang $\mathrm{KQ}$. Celastrol alleviates renal fibrosis by upregulating cannabinoid receptor 2 expression. Cell Death Dis. 2018;9:601.

35. Li R, Li Y, Zhang J, Liu Q, Wu T, Zhou J, Huang H, Tang Q, Huang C, Huang $Y$, et al. Targeted delivery of celastrol to renal interstitial myofibroblasts using fibronectin-binding liposomes attenuates renal fibrosis and reduces systemic toxicity. J Control Release. 2020;320:32-44.

36. Liu BC, Tang TT, Lv LL. How tubular epithelial cell injury contributes to renal fibrosis. Adv Exp Med Biol. 2019;1165:233-52.

37. Arvaniti E, Moulos P, Vakrakou A, Chatziantoniou C, Chadjichristos C, Kavvadas P, Charonis A, Politis PK. Whole-transcriptome analysis of UUO mouse model of renal fibrosis reveals new molecular players in kidney diseases. Sci Rep. 2016;6:26235.

38. Moll S, Chaykovska L, Meier M, Budd DC, Formentini I, Pomposiello S, Prunotto M. Targeting the epithelial cells in fibrosis: a new concept for an old disease. Drug Discov Today. 2013;18:582-91.

39. Nastase MV, Zeng-Brouwers J, Wygrecka M, Schaefer L. Targeting renal fibrosis: mechanisms and drug delivery systems. Adv Drug Deliv Rev. 2018;129:295-307.

40. Eshbach ML, Weisz OA. Receptor-mediated endocytosis in the proximal tubule. Annu Rev Physiol. 2017;79:425-48.

41. Cascao R, Fonseca JE, Moita LF. Celastrol: a spectrum of treatment opportunities in chronic diseases. Front Med (Lausanne). 2017;4:69.

42. Cascao R, Vidal B, Carvalho T, Lopes IP, Romao VC, Goncalves J, Moita $L F$, Fonseca JE. Celastrol efficacy by oral administration in the adjuvantinduced arthritis model. Front Med (Lausanne). 2020;7:455.

43. Shu G, Lu C, Wang Z, Du Y, Xu X, Xu M, Zhao Z, Chen M, Dai Y, Weng Q, et al. Fucoidan-based micelles as P-selectin targeted carriers for synergistic treatment of acute kidney injury. Nanomed Nanotechnol Biol Med. 2021;32:102342.

44. Wang G, Li Q, Chen D, Wu B, Wu Y, Tong W, Huang P. Kidney-targeted rhein-loaded liponanoparticles for diabetic nephropathy therapy via size control and enhancement of renal cellular uptake. Theranostics. 2019;9:6191-208.

45. Luo C, Zhou S, Zhou Z, Liu Y, Yang L, Liu J, Zhang Y, Li H, Liu Y, Hou FF, Zhou L. Wnt9a promotes renal fibrosis by accelerating cellular senescence in tubular epithelial cells. J Am Soc Nephrol. 2018;29:1238-56.

46. Susnik N, Melk A, Schmitt R. Cell aging and kidney repair. Cell Cycle. $2015 ; 14: 3521-2$.
47. Docherty MH, O'Sullivan ED, Bonventre JV, Ferenbach DA. Cellular senescence in the kidney. J Am Soc Nephrol. 2019;30:726-36.

48. Shen L, Zhou J, Wang Y, Kang N, Ke X, Bi S, Ren L. Efficient encapsulation of $\mathrm{Fe}(3) \mathrm{O}(4)$ nanoparticles into genetically engineered hepatitis $\mathrm{B}$ core virus-like particles through a specific interaction for potential bioapplications. Small. 2015;11:1190-6.

49. Shan W, Chen R, Zhang Q, Zhao J, Chen B, Zhou X, Ye S, Bi S, Nie L, Ren $\mathrm{L}$. Improved stable indocyanine green (ICG)-mediated cancer optotheranostics with naturalized hepatitis B core particles. Adv Mater. 2018;30:e1707567.

50. Liu J, Ye Z, Xiang M, Chang B, Cui J, Ji T, Zhao L, Li Q, Deng Y, Xu L, et al. Functional extracellular vesicles engineered with lipid-grafted hyaluronic acid effectively reverse cancer drug resistance. Biomaterials. 2019:223:119475.

51. Chevalier RL, Forbes MS, Thornhill BA. Ureteral obstruction as a mode of renal interstitial fibrosis and obstructive nephropathy. Kidney Int. 2009;75:1145-52.

52. Basnayake K, Ying WZ, Wang PX, Sanders PW. Immunoglobulin light chains activate tubular epithelial cells through redox signaling. J Am Soc Nephrol. 2010;21:1165-73.

53. Villanueva E, Navarro P, Rovira-Rigau M, Sibilio A, Mendez R, Fillat C. Translational reprogramming in tumour cells can generate oncoselectivity in viral therapies. Nat Commun. 2017;8:14833.

54. Shan W, Zhang D, Wu Y, Lv X, Hu B, Zhou X, Ye S, Bi S, Ren L, Zhang X. Modularized peptides modified $\mathrm{HBC}$ virus-like particles for encapsulation and tumor-targeted delivery of doxorubicin. Nanomed Nanotechnol Biol Med. 2018;14:725-34.

55. Feist M, Schwarzfischer P, Heinrich P, Sun X, Kemper J, von Bonin F, PerezRubio P, Taruttis F, Rehberg T, Dettmer K, et al. Cooperative STAT/NF-KB signaling regulates lymphoma metabolic reprogramming and aberrant GOT2 expression. Nat Commun. 2018;9:1514.

\section{Publisher's Note}

Springer Nature remains neutral with regard to jurisdictional claims in published maps and institutional affiliations.
Ready to submit your research? Choose BMC and benefit from:

- fast, convenient online submission

- thorough peer review by experienced researchers in your field

- rapid publication on acceptance

- support for research data, including large and complex data types

- gold Open Access which fosters wider collaboration and increased citations

- maximum visibility for your research: over $100 \mathrm{M}$ website views per year

At BMC, research is always in progress.

Learn more biomedcentral.com/submissions 